
\title{
BResarch S Suare \\ Optimal Ordering Decision and Information Leakage Preference Under Asymmetric Forecast Signal
}

\section{Min Tang}

Chongqing University

\section{Li Jiang}

Chongqing Technology and Business University

\section{Zhiguo Li}

Chongqing Technology and Business University

Hongwu Zhang ( $\nabla$ zhanghongwu@cqu.edu.cn )

Chongqing University

\section{Research Article}

Keywords: Supply chain management, Forecast signal , Asymmetric information , Ordering quantity , Signalling game

Posted Date: April 19th, 2021

DOl: https://doi.org/10.21203/rs.3.rs-412172/v1

License: (1) (1) This work is licensed under a Creative Commons Attribution 4.0 International License. Read Full License 


\title{
Optimal ordering decision and information leakage preference under asymmetric forecast signal
}

\author{
Min Tang ${ }^{1} \cdot$ Li Jiang $^{2} \cdot{\text { Zhiguo } \mathrm{Li}^{3} \text { - Hongwu Zhang }}^{1}$ \\ Received: date / Accepted: date
}

\begin{abstract}
This work investigates the retailer's sourcing decision with or without information leakage and studies the impact of the retailer's inaccurate prediction on supply chain members' preferences for information leakage. To derive the retailers' optimal sourcing quantities under two scenarios, one without and one with information leakage, we formulate a supply chain in which a common manufacturer offers a wholesale price contract to two competing retailers, one of whom (the incumbent) has private prediction information about the market demand, the other one (the entrant) doesn't. Then, we compare the supply chain members' performances, the results show that the manufacturer will prefer no information leakage when the incumbent's forecasting accuracy is high and the forecasting signal is low-type; the incumbent may prefer information leakage under certain conditions; contrary to intuition, the entrant may prefer no information leakage under certain conditions. Meanwhile, we find that the incumbent has an incentive to mimic a low state one by sourcing less product when she forecasts that the market demand
\end{abstract}

Hongwu Zhang $\left({ }^{\bowtie}\right)$

E-mail: zhanghongwu@cqu.edu.cn

Min Tang

E-mail: 20190289001@cqu.edu.cn

Li Jiang

E-mail: 2019603003@mail.ctbu.edu.cn

Zhiguo Li

E-mail: lizhiguo@ctbu.edu.cn

${ }^{1}$ School of Economics and Business Administration, Chongqing University, Chongqing, 400030, China

${ }^{2}$ School of Business Administration, Chongqing Technology and Business University, Chongqing, 400067, China

${ }^{3}$ School of Management Science and Engineering, Chongqing Technology and Business University, Chongqing, 400067, China will be high under the scenario of information leakage, and interestingly, the incumbent will earn less than the entrant when she obtains a low-type forecast signal under no information leakage.

Keywords Supply chain management - Forecast signal · Asymmetric information · Ordering quantity · Signalling game

\section{Introduction}

Within an already fast-moving economy track, the business environment has become increasingly complicated and ever-changing. With the development of information technologies, firms can observe and utilize forecasting information guiding them in operating efficiently. Thus, some large retailing firms have taken advantage of being closer to the end market than other supply chain members (Taylor and Xiao 2010), and have gradually established demand information system to forecast the uncertain market (Fisher et al. 1994). For example, the fast fashion clothing brand Zara adopted a sophisticated information system to forecast market demand to guide its stores' sourcing decisions and warehouse inventory stocking levels (Jiang et al. 2016). Moreover, some well-funded firms have spent considerable money to purchase relevant services or software from thirdparty companies to forecast uncertain demand. For instance, a maker of designer USB flash drives, Mimoco, cooperated with Nielsen Consumer Neuroscience to predict market demand before introducing its products to the market. ${ }^{1}$

1 https://www.nielsen.com/us/en/insights/casestudy/2009/predicting-which-product-designs-drive-thehighest-revenue/ 
However, the firm's prediction is not always completely accurate, which means that the influence of a firm's forecasting accuracy needs to pay attention to when considering its forecast information. According to Dassault Systemes, the industry-leading software firm in planning and optimizing the world's supply chains, more than $50 \%$ of companies doubt the accuracy of their demand prediction information, because forecast results which are based on historical data are not completely accurate. ${ }^{2}$ And the forecasting accuracy indeed has a vital influence on the firm's sourcing decision and performance (Cederlund et al. 2007). Additionally, abilities and expertise in forecasting future market demand are different among the various competing retailers in practice, which means that the competing retailers' forecast information is asymmetric. For instance, in the retail electronics industry, Best Buy had better forecasting ability than Circuit City, which led Circuit City to trail behind its peer retailer Best Buy (Taylor and Xiao 2010). This is mainly because the cost of developing an information forecasting system or outsourcing relevant forecasting services from third parties may be very high for the small, new, or regional businesses, e.g., investing in data mining and data analysis or software.

Under the condition of asymmetric information, direct or indirect information revelation among the competing retailers in a supply chain is universal, which has been proved by many scholars over the past decades. For instance, Anand and Goyal (2009) show that the downstream retailer's order information which can reflect private information is always leaked to its competitor by the upstream manufacturer. They cite an example of Newbury Comics to describe that information leakage among supply chain members with asymmetric information indeed exists in real world. Kong et al. (2013) report that the downstream retailer has an incentive to share its revenue with the upstream manufacturer in order to prevent the upstream manufacturer from leaking information to its competitor. These authors indicate that sensitive information revelation in a supply chain indeed exists especially when the information is asymmetric among the supply chain members, and information leakage in a supply chain will indeed impact the supply chain members' sourcing decisions.

Thereupon, it is worthwhile for us to investigate the retailers' appropriate sourcing decisions when forecasting information is asymmetric and inaccurate under two scenarios, one without and one with information revelation. Under the condition of no information leakage, the retailer who can observe a forecast signal of uncertain demand, i.e., the incumbent, will make her sourcing de-

\footnotetext{
2 https://discover.3ds.com/scenario-based-demandplanning-key-forecasting-accuracy
}

cision according to her forecasting information. While, for the retailer who cannot observe the forecasting signal, e.g., a newly established firm (the entrant), he will make his sourcing decision only according to the common information. Under the condition of information leakage, once the incumbent can signal her private forecasting information through her sourcing quantity, and the entrant can make his sourcing decision according to the incumbent's order information, the two retailers' sourcing decisions may be very different from the condition of no information leakage. This indicates that investigating the downstream retailers' optimal ordering decisions is worthy of the problem.

In what follows, investigating the influence of the downstream retailer's prediction on supply chain members' preferences for information leakage is a valuable research issue as well. Generally, the retailer with private information does not want its information to be leaked, such that the retailer always has to contract with the upstream manufacturer or distort information to ensure that her forecasting information remains confidential. For example, Mishra et al. (2007) illuminate that firms have adequate incentives to distort their information and disclose false information intentionally, and Kong et al. (2013) show that a revenue-sharing contract can protect the informed retailer's information security to realize better performance when forecasting is entirely accurate. However, when the retailer's forecasting information is accurate, the retailer may make no effort to conceal her information which may be wrong. When the incumbent's prediction of market demand is low while real demand is high, if the manufacturer leaks the incumbent's order information, both the two retailers' sourcing quantities will decrease and the manufacturer's income will reduce as a result.

Therefore, some interesting questions are worthy to explore. First, is it always constructive for the manufacturer to leak the incumbent's order information? Second, what is the retailers' optimal ordering decision under the condition of with or without information revelation? Third, is it harmful to the incumbent to leak her information to her competitor? And is it beneficial for the entrant to share information with him?

To describe the above-mentioned issues, we adopted a framework wherein an upstream manufacturer (it) supplies products to two competing retailers to analyse these questions. Based on this framework, to capture the asymmetric and inaccurate information between the two retailers, we assume that the incumbent (she) can observe an inaccurate forecast signal of market demand privately, while the entrant (he) cannot and only knows the prior distribution of market demand. Furthermore, we study the two competing retailers' sourcing quan- 
tities with and without vertical information disclosure. After solving the equilibrium solutions of the two cases, we analyse the supply chain members' preferences with and without information disclosure. From the comparison results, we find some novel insights into the impact of the retailer's forecasting accuracy to the supply chain member.

First, the upstream manufacturer prefers information leakage when the incumbent's forecast signal is high-type, or when the incumbent's forecast signal is low-type and her forecasting accuracy is low, otherwise, it will prefer no information leakage. Second, we find that the entrant can occupy a larger market share under the condition of no information leakage, which means no information leakage may benefit the entrant under certain condition. Furthermore, under the condition of information leakage, once the incumbent can reveal her private forecast signal through her sourcing quantity, the incumbent has an incentive to mimic a low-type one when her forecast signal is high-type. Third, we show that the incumbent will prefer information leakage when she forecasts that the market demand will be high and her forecasting accuracy is low, or when she forecasts that the market demand will be low. Which indicates that, under such a condition, the incumbent has an incentive to voluntarily share her information with the entrant. In addition, we find that the entrant may prefer no information leakage under certain conditions.

The rest of our paper is organized as follows. In Section 2, we briefly summarize the related literature. In Section 3, we describe our model setting. In Section 4, we study the scenario in which the manufacturer does not disclose the incumbent retailer's orders to the entrant retailer. In Section 5, we analyze the retailers' sourcing decisions under prediction information disclosure. In Section 6, we discuss both manufacturer and retailers' preferences. In Section 7, we conclude our main findings and suggestions for future work.

\section{Literature review}

Our paper is primarily related to three research streams on ordering decision, asymmetric forecasting information of market demand, and information revelation and information sharing in a supply chain problems.

\subsection{Ordering decision}

Supply chain members' sourcing decisions, as a key problem in supply chain management, has attracted a lot of scholars' attention (Kim et al. 2011; Jaber et al.
2014; Niu et al. 2019; Khalilpourazari et al. 2019). Chen and Guo (2014) consider a model in which a manufacturer with uncertain supply and two competing retailers to evaluate the combined effects of uncertain supply and retail competition on retailers' sourcing decisions. Jain and Sohoni (2015) examine a supply chain with a supplier and two competing retailers endowed with private information, they study the combined effects of information revelation and moving sequence to retailers' ordering decisions. They find that the downstream retailer can prevent the upstream supplier from leaking its information by using a confidentiality agreement, which will weaken the firm's ability to dampen competition by adjusting sourcing quantity. Yan et al. (2017) consider a supply chain with a common supplier and two competing retailers, they analyse the influence of purchase ways (group buying versus individual purchasing) and moving sequences to the retailers' sourcing decisions under a quantity discount contract. They find that both the two retailers would not prefer group buying even if the wholesale price is lower, and the informed retailer has an incentive to source less to mimic a low state demand if the demand uncertainty is low. Huang et al. (2019) consider a supply chain in which an upstream supplier offers a quantity discount contract to downstream retailer facing uncertain demand, they investigate the optimal contract and the retailer's optimal order quantity under endogenous demand information acquisition.

Although ordering quantity has been investigated under various uncertainty, our paper differs from Chen and Guo (2014), Jain and Sohoni (2015), and Huang et al. (2019), we consider that the downstream retailer's order decision which may be revealed can reflect its private information about the market demand. In contrast to Yan et al. (2017) in what the retailers make their sourcing decisions under a quantity discount contract, our study focuses on the retailers' sourcing decisions under a wholesale price contract.

2.2 Asymmetric forecasting information of the market demand

There have been a number of literature considering asymmetric forecasting information of market demand in a supply chain. Chen and Xiao (2012) consider two competing supply chains, each consisting of one manufacturer and one retailer, only the retailers can observe the asymmetric and inaccurate forecasting information. They find that the competition between the supply chains can lead the downstream retailers have incentive to share their information with their upstream manufacturers, but not with each other. Wu and Zhang 
(2014) assume that the upstream suppliers have asymmetric forecasting information of market demand, the retailers can obtain supplier's private information by sourcing products from them. They find that the retailers prefer sourcing from the supplier with more forecasting information and higher sourcing cost under certain conditions. Jiang et al. (2016) consider a supply chain wherein an upstream manufacturer sells its products through a downstream retailer under a wholesale price contract, the manufacturer has a better demandforecast information than the retailer. They find that the risk-neutral retailer may prefer no-sharing format and the manufacturer may prefers mandatory-sharing format. Nasser and Turcic (2019) consider a supply chain consisting of a manufacturer and a retailer with private forecasting information of the uncertain demand, which incurs a hidden information cost to the manufacturer, and they explore a mechanism to help the manufacturer to weaken or eliminate such an information disadvantage. Meanwhile, Chen and Ozer (2019) assume that the downstream retailers with different forecasting ability of market demand source products from a common supplier, they study a contract which can promote vertical information sharing in a supply chain while avoiding horizontal information leakage between competing retailers.

Compared with the literature we mentioned, although asymmetric forecasting information on market demand in a supply chain has been allowed, in contrast to $\mathrm{Wu}$ and Zhang (2014) and Jiang et al. (2016), we consider the downstream retailer has private forecasting information rather than the upstream supplier. Furthermore, differing from Chen and Xiao (2012) who analyse forecasting information asymmetric between supply chains and Nasser and Turcic (2019) who study information asymmetric between supplier and retailer, we consider forecasting information asymmetric between retailers. Additionally, differing from Chen and Ozer (2019) who consider the retailer only can forecast its own demand, our paper considers the retailer has the forecasting information of the whole market demand.

\subsection{Information revelation and information sharing in a supply chain}

In recent years, information revelation and information sharing has attracted considerable attention. Li (2002) shows that the downstream retailers with private signals have no incentive to voluntarily share their information with the upstream manufacturer for the information leakage will hurt their profits, but the manufacturer can obtain their private information to realize a larger profit by compensating them. Zhang (2002) and
Li and Zhang (2008) consider a supply chain in which a manufacturer provides products to competing retailers with different information. They find that, when the manufacturer's wholesale price can reflect the shared information, revealing the information to the manufacturer may occur a loss to the retailer. Zhu et al (2011) examine a supply chain with one manufacturer and one retailer, both the two players can obtain forecasting information of the market demand independently, the retailer can infer the manufacturer's private forecasting information after observing its wholesale price, thereby the retailer has better forecast than the manufacturer under no information sharing. Meanwhile, many literature consider that the information is revealed confidentially, such as Anand and Goyal (2009), Kong et al. (2013), Yan et al. (2017) and Wang et al. (2019). All of them consider a framework in which a supplier and two competing downstream retailers with asymmetric information of market demand, and the demand information are shared through sourcing quantity in a supply chain. Anand and Goyal (2009) find that, under a wholesale price contract, the upstream supplier always has an incentive to disclose the retailer's order information reflecting private demand information. Kong et al. (2013) show that the revenue sharing contract can help the retailer to prevent the upstream supplier from leaking its order information reflecting private information to its competitor. Differing from Anand and Goyal (2009) and Kong et al. (2013), Yan et al. (2017) consider a quantity discount contract, thereby the order information can be revealed directly between the retailers. Wang et al. (2019) show that whether the supplier leaks information is affected by multiple factors when the downstream retailers are asymmetric.

While many papers investigate information revelation and information sharing, different from Li (2002) and Yan et al. (2017), this paper considers the information is revealed confidentially and indirectly. Further, differing from Zhang (2002), Li and Zhang (2008) and Zhu et al. (2011), we suppose that the demand information is shared by the retailer's sourcing quantity. Moreover, Anand and Goyal (2009), Kong et al. (2013) and Wang et al. (2019) consider that one of the two retailers can observe accurate information on market demand, differently, this paper considers that the information the retailer observed is inaccurate which will be closer to reality.

\section{Model framework}

We consider a supply chain in which two competing retailers source products from a common manufacturer 
and then sell all the merchandise to the end-user market. The retailers are asymmetric with demand prediction information, so we distinguish the one with information advantages as the incumbent (she) and the other one as the entrant (he). For the reminder of our paper, we use $i, e$, and $m$ to denote the incumbent, the entrant, and the manufacturer respectively. All supply chain members are risk-neutral and try to maximize their own expected profit.

The retailers are engaged in quantity competition so that the market demand is set to follow a linear, downward-sloping function, $P=A-Q$, where $P$ is the market clearance price, $A$ is the market potential, and $Q=q_{i}+q_{e}$ is the total quantity which is provided in the market. Given the market potential, the more products are provided in the market, the lower price will incur. Specifically, $q_{i}$ and $q_{e}$ are the sourcing quantities of the incumbent and the entrant respectively, which are provided to consumers at the market-clearing price. Additionally, without loss of generality, we suppose that the market potential $A$ is large enough such that the retail price is always positive. The inverse demand function is widely used in capturing quantity competition in a game model, such as Anand and Goyal (2009), Kong et al. (2013), Niu et al. (2019) and Li et al. (2020), so it is also adopted in our model.

The market demand is always uncertain and the two retailers have asymmetric information about it. We assume that the market potential $A$ fluctuates within a binary distribution and there are two possible states for the market potential: either high type (denoted as $A_{H}$ ) or low type (denoted as $A_{L}$ ). Suppose $A_{H}$ has the value $(1+\delta) \bar{A}$ with probability $\theta \in(0,1)$ and $A_{L}$ has the value $(1-\delta) \bar{A}$ with probability $1-\theta$, where $\bar{A}$ refers to the average market potential and $\delta \in(0,1)$ can be considered to be the level of demand uncertainty, as the parameter $\delta$ increases, the level of demand uncertainty increases. It noteworthy that the incumbent has better information about the market potential $A$ than the entrant, but the ex ante distribution of $A$ is common knowledge to all supply chain members. For example, the firms' information about the market demand has a positive correlation with their existing time, so that the incumbent knows more about the market demand by analysing the historical data collected by her, but the entrant just has the prior distribution of the market demand. Similar assumption have been widely adopted by previous studies such as Jiang et al. (2016), Yan et al. (2017) and Wang et al. (2019).

To characterize the incumbent's information advantage, we assume that only the incumbent can observe a prediction of the market potential which is denoted as $s$. For the convenience to discuss, we assume that the forecast of demand also has two possible values: $s=l$ and $s=h$. Let the parameter $\rho \in[0,1]$ measures the accuracy of the incumbent's prediction, note that, the prediction is imperfect as long as $0<\rho<1$. Therefore, $\rho=1$ indicates that the forecast is entirely accurate, whereas $\rho=0$ means that the forecast is totally inaccurate, that means, the larger the $\rho$ is, the more reliable the prediction information is. Furthermore, we suppose that the incumbent's forecast is unbiased, such that the unconditional probability of a prediction indicating any demand state is equal to the prior probability of that state (i.e., $\operatorname{Pr}\left(A_{H}\right)=\operatorname{Pr}(h)$ and $\left.\operatorname{Pr}\left(A_{L}\right)=\operatorname{Pr}(l)\right)$. Similar assumptions can be found in Iyer et al. (2007) and Jiang et al. (2016). Obviously, one can easily derive the conditional probabilities: $\operatorname{Pr}\left(h \mid A_{L}\right)=\operatorname{Pr}\left(A_{H} \mid l\right)=\theta(1-\rho), \operatorname{Pr}\left(h \mid A_{H}\right)=$ $\operatorname{Pr}\left(A_{H} \mid h\right)=\theta+(1-\theta) \rho, \operatorname{Pr}\left(l \mid A_{H}\right)=\operatorname{Pr}\left(A_{L} \mid h\right)=$ $(1-\theta)(1-\rho)$ and $\operatorname{Pr}\left(l \mid A_{L}\right)=\operatorname{Pr}\left(A_{L} \mid l\right)=(1-\theta)+\theta \rho$. Thus, for any $\rho \in(0,1)$, the imperfect forecast signal always contributes to improve the demand information of the retailers.

There are two scenarios for comparison in our paper: no information leakage and information leakage. Under the condition of no information leakage, we consider that the upstream manufacturer doesn't leak the incumbent's order information reflecting the private forecasting information to the entrant. While under the condition of information leakage, we consider that the upstream manufacturer always leaks the incumbent's order information to the entrant. To facilitate the discussion, we use the superscript $N L$ and $L$ to refer to the two scenarios. The sequence of events is as follows: first, the incumbent decides her sourcing quantity $q_{i}$ after observing a forecast signal $s$; second, the upstream manufacturer leaks the incumbent's order information to the entrant under the scenario of information leakage, and does not leak under the scenario of no information leakage; third, the entrant decides his sourcing quantity $q_{e}$; then, "nature" determines the actual market demand state; finally, both the two retailers' products are provided to the end-users market, and the market clearing price is realized. Furthermore, when we're deriving the two retailers' optimal sourcing quantities, to remain our focus, we normalize that $\omega$ is equal to zero.

\section{Sourcing decisions under no information leakage (NL)}

We first consider the scenario of no information leakage, under such a condition, the manufacturer never leaks the incumbent's order information to the entrant, which can be recognized as a Nash game. Based on the above 
model setting, given the forecast signal state $s \in\{l, h\}$, the incumbent's expected profit function is as follows:

$\mathrm{E}\left[\Pi_{i s}^{N L}\left(q_{i s}^{N L} \mid s\right)\right]=\left(\mathrm{E}[A \mid s]-q_{i s}^{N L}-q_{e}^{N L}\right) q_{i s}^{N L}$,

and the entrant's expected profit can be written as:

$$
\begin{aligned}
\mathrm{E}\left[\Pi_{e}^{N L}\left(q_{e}^{N L}\right)\right]= & \sum_{s \in\{l, h\}} \operatorname{Pr}(s)(\mathrm{E}[A \mid s] \\
& \left.-q_{i s}^{N L}-q_{e}^{N L}\right) q_{e}^{N L},
\end{aligned}
$$

where $\mathrm{E}[A \mid s]=A_{H} \operatorname{Pr}\left(A_{H} \mid s\right)+A_{L} \operatorname{Pr}\left(A_{L} \mid s\right)$. For convenience, we define $\tau \equiv 1+\delta(2 \theta-1)(1-\rho)$ to indicate the average of expected demand, meanwhile, we use $\Delta \equiv \delta \rho$ to measure the difference of expected demand between $h$-state and $l$-state, this means that the expected demand would change more as $\Delta$ increasing. The equilibrium outcome under this scenario is summarized in Lemma 1, more calculative details and analyses are given in the appendix.

Lemma 1 If the upstream manufacturer never leaks the incumbent's information to the entrant, the equilibrium outcomes are listed as follows. Note that, we acquiesce $0<\Delta \leq \frac{\tau}{1+\theta}$ to ensure that the retailers' sourcing quantities are non-negative.

(1) The incumbent's optimal orders are:

$$
q_{i}^{N L}= \begin{cases}\frac{[\tau+\Delta+(1-\theta) \Delta] \bar{A}}{3}, & \text { if } s=h \\ \frac{(\tau-\Delta-\theta \Delta) \bar{A}}{3}, & \text { if } s=l .\end{cases}
$$

(2) The entrant's optimal sourcing quantity is:

$$
q_{e}^{N L}=\frac{(\tau-\Delta+2 \theta \Delta) \bar{A}}{3} .
$$

(3) The incumbent's maximal expected profit is:

$$
\mathrm{E}\left[\Pi_{i}^{N L}\right]= \begin{cases}\frac{[\tau+\Delta+(1-\theta) \Delta]^{2} \bar{A}^{2}}{9}, & \text { if } s=h \\ \frac{(\tau-\Delta-\theta \Delta)^{2} \bar{A}^{2}}{9}, & \text { if } s=l .\end{cases}
$$

(4) The entrant's maximal expected profit is:

$$
\mathrm{E}\left[\Pi_{e}^{N L}\right]=\frac{(\tau-\Delta+2 \theta \Delta)^{2} \bar{A}^{2}}{9} .
$$

In the case of the manufacturer never leaks the incumbent's order information to the entrant, the incumbent can exclusively enjoy the information advantage in terms of market demand prediction. For the incumbent, she just needs to make her sourcing decisions which only rely on the forecast signal she observed, and no longer worries about the manufacturer disclosing her information. For the entrant, without information leakage, he cannot infer any information of uncertain demand and only has the ex ante distribution of the market potential. Under such a condition, we explore two retailers' expected profits in Corollary 1.
Corollary 1 When the manufacturer never discloses the incumbent's orders, the incumbent can earn more if $s=h$, whereas the entrant can earn more if $s=l$.

Corollary 1 indicates that, for any $\Delta \in\left(0, \frac{\tau}{1+\theta}\right]$, the two retailers' expected profits follows

$\mathrm{E}\left[\Pi_{i h}^{N L}\right]>\mathrm{E}\left[\Pi_{e}^{N L}\right]>\mathrm{E}\left[\Pi_{i l}^{N L}\right]$,

which illuminates that the incumbent can earn more than the entrant if her forecast signal is high state. While if the forecast signal is low, the entrant can earn more than the incumbent. We can understand such a result from the two retailers' sourcing decisions. For the incumbent, if she observes a low-state forecast signal about the market demand, she would decrease her order to maintain profitability. For the entrant without any forecast information, he can only place a same sourcing quantity regardless of the signal state. Therefore, when the forecast signal is low-state, only the incumbent reduces her orders, since that, if the incumbent spots a low-state signal in advance, she would like to share her sourcing quantity to cause the entrant to decrease his orders as well, thereby, the market clearance price will increase, and the incumbent's profit will increase too. More details of the incumbent's voluntarily sharing preference are discussed in Section 6.2.

\section{Sourcing decisions under information leakage (L)}

In this section, we suppose that the upstream manufacturer always leaks the incumbent's ordering quantity reflecting her private forecasting information to the entrant. Thereby, the entrant can make his sourcing decision according to the incumbent's orders the manufacturer leaked. The two retailers' expected profits are

$\mathrm{E}\left[\Pi_{i}^{L}\left(q_{i s}^{L} \mid s\right)\right]=\left(\mathrm{E}[A \mid s]-q_{i s}^{L}-q_{e}^{L}\right) q_{i s}^{L}$

and

$\mathrm{E}\left[\Pi_{e}^{L}\left(q_{e}^{L} \mid q_{i s}^{L}\right)\right]=\left[\sum_{s \in\{l, h\}} \operatorname{Pr}\left(s \mid q_{i}\right) \mathrm{E}[A \mid s]-q_{i s}^{L}-q_{e}^{L}\right] q_{e}^{L}$

severally. Similarly, we use $\tau \equiv 1+\delta(2 \theta-1)(1-\rho)$ to indicate the average of expected demand, and $\Delta \equiv \delta \rho$ to measure the interval of $\mathrm{E}[A \mid h]$ and $\mathrm{E}[A \mid l]$.

Once the incumbent realizes that the manufacturer may leak her order quantity, she will strategically readjust her order to maximize her expected profit. Due to the entrant can deduce the demand forecast type indirectly, the incumbent will think over the entrant's action before she places her sourcing orders. Therefore, a 
signalling game emerges, as common knowledge in such a game, two types of pure strategy perfect Bayesian equilibria arise: the separating equilibrium and the pooling equilibrium. We discuss the two equilibria in Section 5.1 and Section 5.2 separately. The two superscripts $S$ and $P$ are used to indicate the two kinds of equilibrium respectively.

\subsection{The separating equilibrium}

The separating equilibrium supposes that the incumbent makes different sourcing decisions for each forecast state, thereby the entrant can infer the forecast state after obtaining the incumbent's sourcing quantity. In such an equilibrium, for the incumbent, if the forecast signal is $l$, her sourcing quantity is $q_{i}=q_{i l}^{L S}$; if the forecast signal is $h$, her sourcing quantity is $q_{i}=q_{i h}^{L S}>q_{i l}^{L S}$.

For the entrant, according to the incumbent's sourcing quantity, he can infer the forecasting demand state and update his belief in terms of the market demand later. It is reasonable for the entrant to deduce that the incumbent will purchase more when she observes a high signal, such that, the entrant's belief system with a threshold quantity can be given by:

$\operatorname{Pr}(s=h)= \begin{cases}1, & \text { if } q_{i}>q_{i l}^{L S} \\ 0, & \text { if } q_{i} \leq q_{i l}^{L S},\end{cases}$

which indicates that the entrant believes that the demand forecast is high if the incumbent retailer's sourcing quantity $q_{i}>q_{i l}^{L S}$ and low otherwise.

Next, we discuss the incentive compatibility constraints of the separating equilibrium to ensure that the incumbent is willing to separate under each type of forecast. The constraints can be indicated as the follows:

$$
\left\{\begin{array}{l}
\max _{q_{i}} \mathrm{E}\left[\Pi_{i}\left(q_{i}>q_{i l}^{L S} \mid h\right)\right] \geq \max _{q_{i}} \mathrm{E}\left[\Pi_{i}\left(q_{i} \leq q_{i l}^{L S} \mid h\right)\right] \\
\max _{q_{i}} \mathrm{E}\left[\Pi_{i}\left(q_{i} \leq q_{i l}^{L S} \mid l\right)\right] \geq \max _{q_{i}} \mathrm{E}\left[\Pi_{i}\left(q_{i}>q_{i l}^{L S} \mid l\right)\right]
\end{array}\right.
$$

The LHS of those inequation constraints are the incumbent's expected profits under the equilibrium which are greater than the RHS off-equilibrium profits, such that the separating equilibrium is realized. Then, we can find the incumbent's possible motivation by analysing her expected profits under different conditions, which are presented in Lemma 2.

Lemma 2 Given $\delta, \rho, \theta$ and $s$, the incumbent who observed a high-type forecast signal has an incentive to mimic a low type one, while the low-type incumbent does not.
Lemma 2 indicates that the second constraint always holds, and since only when the incumbent's prediction of the demand is high type, she has an intention to mimic a low-state one, due to the higher profit she can obtain. However, the second constraint above does not bind, this shows that the incumbent will never have an incentive to pretend to be a high-state one when her prediction of the market demand is low. We can further understand the incumbent's incentive by analysing the inverse demand function, $P=A-Q$, which illuminates that the market demand clearing price will increase if the total sourcing quantity of the two retailers decreases. Therefore, when the incumbent observed a high-state forecast signal in advance, she would mimic a low-state one to induce the entrant to purchase less to realize a higher market clearing price. Lemma 3 summarizes the separating equilibrium outcome. Here again, more calculative details are given in the appendix.

Lemma 3 For any $\Delta>0$, under the condition of information leakage, the separating equilibrium exists:

(1) The optimal sourcing quantities of the incumbent are:

$$
q_{i}^{L S}= \begin{cases}\frac{(\tau+\Delta) \bar{A}}{2} & \text { if } s=h, \\ \frac{(\tau-\Delta) \bar{A}}{2}(1-\mathcal{D}) & \text { if } s=l \text { and } 0<\Delta<\frac{\tau}{2}, \\ \frac{(\tau-\Delta) \bar{A}}{2} & \text { if } s=l \text { and } \frac{\tau}{2} \leq \Delta<1 .\end{cases}
$$

(2) The optimal sourcing quantities of the entrant are:

$$
q_{e}^{L S}= \begin{cases}\frac{(\tau+\Delta) \bar{A}}{4} & \text { if } \operatorname{Pr}(s=h)=1, \\ \frac{(\tau-\Delta) \bar{A}}{4}(1+\mathcal{D}) & \text { if } \operatorname{Pr}(s=h)=0 \text { and } 0<\Delta<\frac{\tau}{2}, \\ \frac{(\tau-\Delta) \bar{A}}{4} & \text { if } \operatorname{Pr}(s=h)=0 \text { and } \frac{\tau}{2} \leq \Delta<1 .\end{cases}
$$

(3) The maximal expected profits of the incumbent are:

$\mathrm{E}\left[\Pi_{i}^{L S}\right]= \begin{cases}\frac{(\tau+\Delta)^{2} \bar{A}^{2}}{8} & \text { if } s=h, \\ \frac{(\tau-\Delta)^{2} \bar{A}^{2}}{8}\left(1-\mathcal{D}^{2}\right) & \text { if } s=l \text { and } 0<\Delta<\frac{\tau}{2}, \\ \frac{(\tau-\Delta)^{2} \bar{A}^{2}}{8} & \text { if } s=l \text { and } \frac{\tau}{2} \leq \Delta<1 .\end{cases}$

(4) The maximal expected profits of the entrant are:

$$
\mathrm{E}\left[\Pi_{e}^{L S}\right]=\left\{\begin{array}{lr}
\frac{(\tau+\Delta)^{2} \bar{A}^{2}}{16} & \text { if } \operatorname{Pr}(s=h)=1, \\
\frac{(\tau-\Delta)^{2} \bar{A}^{2}}{16}(1+\mathcal{D})^{2} & \text { if } \operatorname{Pr}(s=h)=0 \\
\frac{(\tau-\Delta)^{2} \bar{A}^{2}}{16} & \text { and } 0<\Delta<\frac{\tau}{2}, \\
& \text { if } \operatorname{Pr}(s=h)=0 \\
& \text { and } \frac{\tau}{2} \leq \Delta<1 .
\end{array}\right.
$$

Here $\mathcal{D} \equiv \frac{2[\sqrt{(\tau+2 \Delta) \Delta}-2 \Delta]}{\tau-\Delta} \in(0,1)$ represents the forecast information distorting degree by signaling, where $0<\Delta<\frac{\tau}{2}$. 
In the separating equilibrium, the entrant can infer the type of the forecasting demand signal by analysing the incumbent's sourcing quantity which is disclosed by the upstream manufacturer. Thus, both the two retailers have the forecast information, the difference is that the incumbent's forecast information is first-hand while the entrant's forecast signal is obtained indirectly. Since the upstream manufacturer always discloses the incumbent's orders, the incumbent will decide whether to mimic, and prefer to make sourcing decision strategically in order to make advantage of her prediction information in terms of market demand.

Since the upstream manufacturer always discloses the incumbent's sourcing quantity to the entrant, the incumbent needs to take into account the entrant's sourcing decision before making her decision. If $0<\Delta<$ $\frac{\tau}{2}$, which means the difference between $\mathrm{E}\left[A_{H} \mid h\right]$ and $\mathrm{E}\left[A_{L} \mid l\right]$ is small, the incumbent needs to decrease her sourcing quantity, so that the entrant can recognize her signal accurately. Specifically, if the incumbent observed a high-state forecast signal, for any $0<\Delta<\frac{\tau}{2}$, she would reduce her sourcing quantity even lower than the low-state optimal purchasing quantity to separate with the high-state one, which could signal the correct information to the entrant. However, when $\frac{\tau}{2} \leq \Delta<1$, that is, when the expected demands between the highstate and the low-state are far apart, the cost of information distorting increases, for these reasons, the incumbent will have no incentive to mimic under such a condition, which means that the low-state forecast signal could spontaneously separate from the high-state one.

For the entrant, he will deduce the forecast signal state by analysing the incumbent's orders the upstream manufacturer disclosed, then he will utilize the forecast information he inferred to adjust his orders to maximize his expected profit. When $q_{i}>q_{i l}^{L S}$, the entrant will believe that the incumbent's forecasting demand must be high, and he will implement a high sourcing strategy. When $0<\Delta<\frac{\tau}{2}$ and the interval between $\mathrm{E}\left[A_{H} \mid h\right]$ and $\mathrm{E}\left[A_{L} \mid l\right]$ is small, so it is difficult for the entrant to discriminate the forecast signal state which makes the incumbent reducing her sourcing quantity to separate the low type one from the high type. Thus, when the entrant observes that the incumbent's sourcing quantity is $\frac{(\tau-\Delta) \bar{A}}{2}(1-\mathcal{D})$, he will believe that the forecast signal is low-state, and implement a low type sourcing strategy. When $\frac{\tau}{2} \leq \Delta<1, \mathrm{E}\left[A_{H} \mid h\right]$ and $\mathrm{E}\left[A_{L} \mid l\right]$ are far apart, the incumbent's information distorting cost becomes large and the incumbent will not mimic, the entrant will not worry about the incumbent deceiving him, and he could obtain the right forecast signal no matter what the signal type is.

\subsection{The pooling equilibrium}

In a pooling equilibrium, the incumbent would like to source a same quantity, no matter what the signal type she observed is, thus, the entrant cannot infer the incumbent's forecast type even though the upstream manufacturer leaks the incumbent's sourcing quantity to him. In detail, for the incumbent, she would order a same quantity under both signal types, owing to she does not want the entrant to have any forecast information under the condition of disclosure. The entrant's belief system with a threshold can be given as follows:

$\operatorname{Pr}(s=h)= \begin{cases}1, & \text { if } q_{i}>q_{i}^{L P} \\ \theta, & \text { if } q_{i} \leq q_{i}^{L P}\end{cases}$

The entrant's preference depends on his deduction, once the incumbent orders the same quantity, the entrant cannot deduce the type of forecast signal, he would source a same quantity too. The incumbent would like to pool if ans only if the following constraints exist:

$$
\left\{\begin{array}{l}
\max _{q_{i}} \mathrm{E}\left[\Pi_{i}\left(q_{i} \leq q_{i}^{L P} \mid h\right)\right]=\mathrm{E}\left[\Pi_{i}\left(q_{i}=q_{i}^{L P} \mid h\right)\right], \\
\max _{q_{i}} \mathrm{E}\left[\Pi_{i}\left(q_{i} \leq q_{i}^{L P} \mid l\right)\right]=\mathrm{E}\left[\Pi_{i}\left(q_{i}=q_{i}^{L P} \mid l\right)\right], \\
\max _{q_{i}} \mathrm{E}\left[\Pi_{i}\left(q_{i} \leq q_{i}^{L P} \mid h\right)\right] \geq \max _{q_{i}} \mathrm{E}\left[\Pi_{i}\left(q_{i}>q_{i}^{L P} \mid h\right)\right], \\
\max _{q_{i}} \mathrm{E}\left[\Pi_{i}\left(q_{i} \leq q_{i}^{L P} \mid l\right)\right] \geq \max _{q_{i}} \mathrm{E}\left[\Pi_{i}\left(q_{i}>q_{i}^{L P} \mid l\right)\right] .
\end{array}\right.
$$

The following proposition shows the pooling equilibrium. More details and technical analyses are given in the appendix too.

Lemma 4 When $\frac{(1-\theta) \tau}{2-3 \theta-\theta^{2}}<\Delta<\frac{\tau}{2+\theta}$, a pooling equilibrium exists and we show it below:

(1) The incumbent orders:

$$
q_{i}^{L P}=\frac{(\tau-\Delta-2 \theta \Delta) \bar{A}}{2} .
$$

(2) The entrant orders:

$$
q_{e}^{L P}=\frac{(\tau-\Delta+6 \theta \Delta) \bar{A}}{4} .
$$

(3) The expected profits of the incumbent are:

$$
\mathrm{E}\left[\Pi_{i}^{L P}\right]= \begin{cases}\frac{(\tau-\Delta-2 \theta \Delta)^{2}+8 \Delta(\tau-\Delta-2 \theta \Delta) \bar{A}^{2}}{(\tau-\Delta-2 \theta \Delta)^{2} \bar{A}^{2}} & \text { if } s=h, \\ \frac{(\tau}{8} & \text { if } s=l .\end{cases}
$$

(4) The expected profit of the entrant is:

$$
\mathrm{E}\left[\Pi_{e}^{L P}\right]=\frac{(\tau-\Delta+6 \theta \Delta)^{2} \bar{A}^{2}}{16} .
$$


As the Lemma 4 illuminated, the pooling equilibrium exists when $\frac{(1-\theta) \tau}{2-3 \theta-\theta^{2}}<\Delta<\frac{\tau}{2+\theta}$. From Lemma 4, we can find that both the two states of the incumbent's sourcing quantity are decreasing. We can understand such a condition from the inverse demand function, $P=A-Q$. For the incumbent, reducing her sourcing quantity can increase the market clearing price, which motives her to decrease her sourcing quantity to realize a higher profit under some specific condition, even though the prediction of demand is high. By the same token, the incumbent will have an similar incentive to decrease her sourcing quantity when she predicts a lowtype market demand. For the entrant, both types of incumbents source a same quantity, so that he cannot infer out the types of forecasting signal but only orders a same quantity too. Note that, differing from the condition of the upstream manufacturer never leaks, under pooling, the entrant can update his belief according to the incumbent's sourcing quantity the manufacturer disclosed. Comparing to the profit of the entrant without information leakage, the entrant's income reduces, which is lead by the incumbent's strategic sourcing decision. Therefore, in some cases, without any information of market demand may be constructive for the entrant.

\subsection{Equilibrium outcome}

Based on the results we solved above, for the incumbent, we find that there are multiple equilibria in our model setting when $\frac{(1-\theta) \tau}{2-3 \theta-\theta^{2}}<\Delta<\frac{\tau}{2+\theta}$, this indicates that we need to find the optimal equilibrium outcome. So that, we use LMSE (Lexicographically Maximum Sequential Equilibrium) concept to find such a unique outcome (see Mailath et al. 1993), the LMSE concept has been widely used as one of the multiple equilibria selection criterion (such as Guo and Zhang 2017; Jiang et al. 2016, 2020), thus, it is also adapted in our setting. The unique and optimal equilibrium result is summarized in Proposition 1, the relevant proof is given in appendix.

Proposition 1 Under information leakage, the equilibrium outcome is as follows:

- When $\Delta \in\left(\frac{(1-\theta) \tau}{2-3 \theta-\theta^{2}}, \frac{\tau}{2+\theta}\right)$, the incumbent prefers separating equilibrium and choosing $q_{i}=\frac{(\tau+\Delta) \bar{A}}{2}$ if $s=h$ and $q_{i}=\frac{(\tau-\Delta) \bar{A}}{2}(1-\mathcal{D})$ if $s=l$ as her sourcing quantity separately.

-When $\Delta \notin\left(\frac{(1-\theta) \tau}{2-3 \theta-\theta^{2}}, \frac{\tau}{2+\theta}\right)$, there is only separating equilibrium.

As the Proposition 1 illuminates, there indeed exists the unique choice of equilibrium by using the LMSE concept we refined. We compare the l-type incumbent's two kinds of pure-strategy expected profit, and find that $\mathrm{E}\left[\Pi_{i l}^{L S}\right]>\mathrm{E}\left[\Pi_{i l}^{L P}\right]$ always holds when $\frac{(1-\theta) \tau}{2-3 \theta-\theta^{2}}<\Delta<$ $\frac{\tau}{2+\theta}$, that is, if the prediction of market demand is lowtype, the separating equilibrium payoff is larger than the pooling equilibrium's. Which means that the separating equilibrium $l$-dominates the pooling equilibrium when $\frac{(1-\theta) \tau}{2-3 \theta-\theta^{2}}<\Delta<\frac{\tau}{2+\theta}$, under such a condition, the incumbent will prefer separating equilibrium and implement the separating sourcing strategy when there are multiple equilibria to choose from. Otherwise, there is only separating equilibrium, and the incumbent will choose $q_{i}=\frac{(\tau+\Delta) \bar{A}}{2}$ if $s=h, q_{i}=\frac{(\tau-\Delta) \bar{A}}{2}(1-\mathcal{D})$ if $s=l$ and $0<\Delta<\frac{\tau}{2}, q_{i}=\frac{(\tau-\Delta) \bar{A}}{2}$ if $s=l$ and $\frac{\tau}{2} \leq \Delta<1$ as her sourcing quantity.

\section{Analysis}

In this section, we compare the expected profits of the three game players to find the effect of the downstream retailer's inaccurate prediction on each party's preference for information revelation. We discuss the manufacturer's preference in Section 6.1, and the two retailers' preferences in Section 6.2 and Section 6.2 separately. The superscripts $L$ and $N L$ are used to denote the two scenarios severally.

\subsection{The manufacturer's performance comparison}

In this section, we compare the manufacturer's ex post profits under two different scenarios to find the influence of the downstream retailer's inaccurate forecasting on the manufacturer's preference for information leakage. When we discussing the manufacturer's best decision, for simplify, we will assume that the manufacturer's total cost of per unit product is equal to zero and the wholesale price $\omega$ is exogenous, this does not affect the major results and allows us to focus on the manufacturer's preference. Meanwhile, we suppose that the market potential is large enough to make sure that the manufacturer's revenue is always positive. Due to the manufacturer is a profit maximizer, he would prefer the most beneficial information management strategy, we show such a result in the following proposition.

Proposition 2 Comparing the manufacturer's performance, we have:

(1) If $s=h, \mathrm{E}\left[\Pi_{m}^{L}\right]>\mathrm{E}\left[\Pi_{m}^{N L}\right]$;

(2) If $s=l, \mathrm{E}\left[\Pi_{m}^{L}\right]>\mathrm{E}\left[\Pi_{m}^{N L}\right]$ for $\rho<\frac{1-\delta+2 \theta \delta}{6 \theta \delta}$, otherwise, $\mathrm{E}\left[\Pi_{m}^{L}\right]<\mathrm{E}\left[\Pi_{m}^{N L}\right]$. 
Comparing the two scenarios, we find that, given any $\theta, \delta$ and $\rho$, the downstream retailer's inaccurate prediction will indeed impact the manufacturer's preference for information leakage. The Proposition 2 shows that the manufacturer's profit under information leakage is larger than no information leakage for any forecasting accuracy if the incumbent forecasts that the market demand will be high, which indicates that the manufacturer always prefers information leakage for any $\rho$ when $s=h$. This condition no longer holds when $s=l$. It is interesting to find that the downstream retailer's forecasting accuracy will influence the manufacturer's preference. From the Proposition 2, we find that the manufacturer's expected profit under information leakage is higher than no information leakage if the incumbent's forecasting accuracy is smaller than a

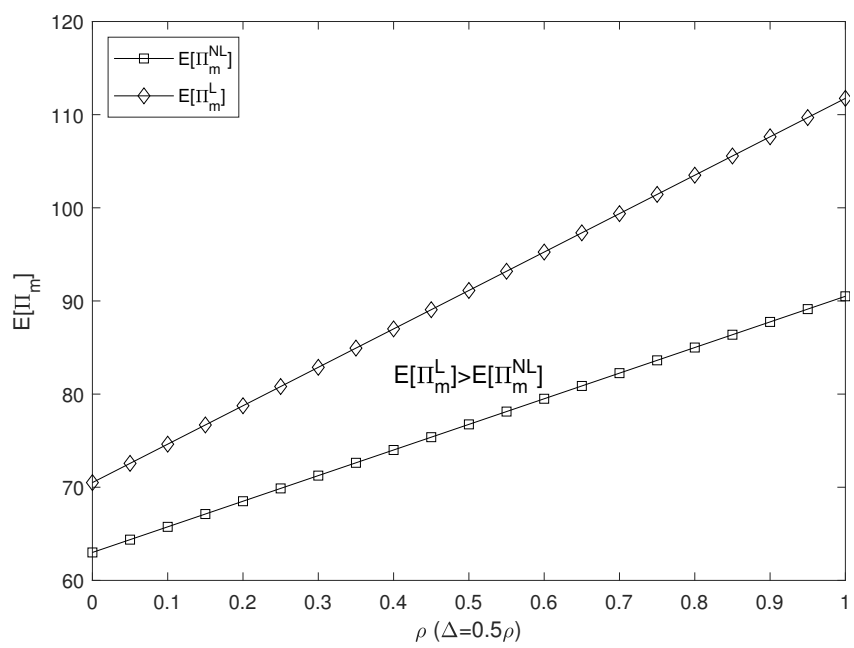

(a) An example of the manufacturer's preference when $s=h$.

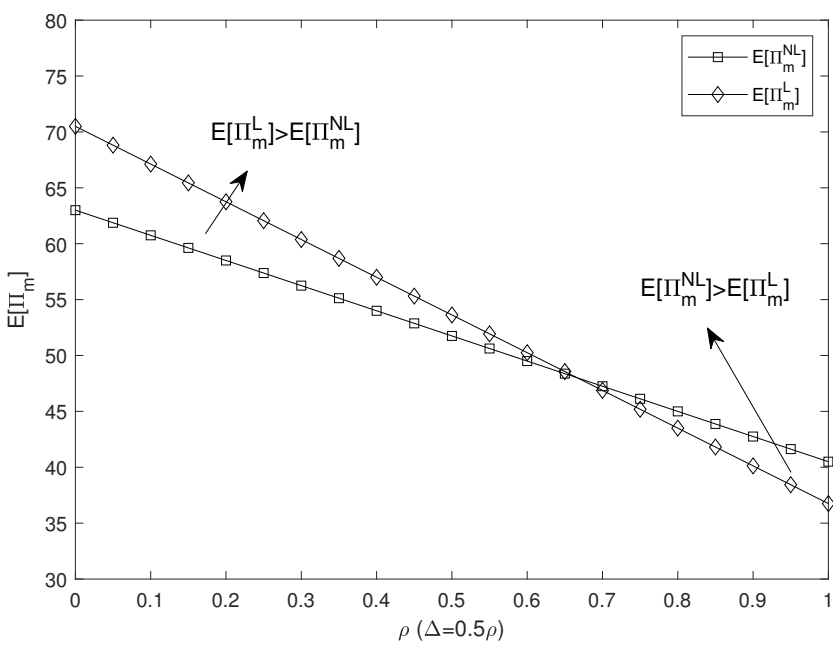

(b) An example of the manufacturer's preference when $s=l$.

Fig. 1 Example of a two-part figure with individual subcaptions showing that information leakage may hurt the manufacturer's profit. threshold when the incumbent forecasts that the market demand will be low, which means that the manufacturer will prefer information leakage if $\rho$ is low when $s=l$. Otherwise, the manufacturer's expected profit under no information leakage will larger than information, which indicates that information leakage may hurt its profit if the incumbent's forecasting accuracy is high when $s=l$.

We illustrate this proposition in Figure 1, the manufacturer's expected profit $\mathrm{E}\left[\Pi_{m}\right]$ as a function of $\rho$ for $\omega=1, \bar{A}=100, \delta=0.5$ and $\theta=0.45$. We use the solid line with diamond illustrating the manufacturer's profit under leakage and the solid line with square illustrating its profit without information leakage. Then we can visually find that the manufacturer prefers information leakage when $s=h$, but it will prefer information leakage if $\rho$ is small when $s=l$.

\subsection{The incumbent's performance comparison}

In this part, we compare the profits of the incumbent under different situations to find her preference for information leakage. Note that, without loss of generality, we normalize that the wholesale price $\omega$ is equal to zero. Furthermore, when $s=l$, we assume $\Delta<\frac{\tau}{1+\theta}$ (i.e., $\left.\rho<\frac{1-\delta+2 \theta \delta}{3 \theta \delta}\right)$ to ensure that the incumbent's sourcing quantity is non negative. The outcome is summarized in Proposition 3.

Proposition 3 The outcome of the incumbent's performance comparison is as follows:

(1) When $s=l, \mathrm{E}\left[\Pi_{i}^{L}\right]>\mathrm{E}\left[\Pi_{i}^{N L}\right]$;

(2) When $s=h$, her preference is impacted by her forecasting accuracy:

$$
\begin{aligned}
& \text { ○f } \theta \geq \frac{4 \sqrt{2}-3}{2 \sqrt{2}}, \mathrm{E}\left[\Pi_{i}^{L}\right]>\mathrm{E}\left[\Pi_{i}^{N L}\right] ; \\
& \text { ○ If } \theta<\frac{4 \sqrt{2}-3}{2 \sqrt{2}}, \mathrm{E}\left[\Pi_{i}^{L}\right]>\mathrm{E}\left[\Pi_{i}^{N L}\right] \text { when } \rho< \\
& \frac{(3-2 \sqrt{2})(1-\delta+2 \theta \delta)}{6(\sqrt{2}-1)(1-\theta) \delta}, \text { and vice versa. }
\end{aligned}
$$

When the incumbent forecasts that the market demand will be low (i.e., $s=l$ ), given any forecasting accuracy, $\theta$ and $\delta$, once her sourcing quantity is nonnegative, the incumbent will obtain a larger profit under information leakage than no information leakage, which shows that the incumbent prefers information leakage when $s=l$. From the Proposition 2 and Proposition 3, we can find that both the manufacturer and the incumbent will prefer information leakage when $\rho<\frac{1-\delta+2 \theta \delta}{6 \theta \delta}$, under such a condition, information leakage will realize a win-win condition between the manufacturer and the incumbent. However, if $\frac{1-\delta+2 \theta \delta}{6 \theta \delta} \leq \rho<\frac{1-\delta+2 \theta \delta}{3 \theta \delta}$, the manufacturer prefers no information leakage, while 


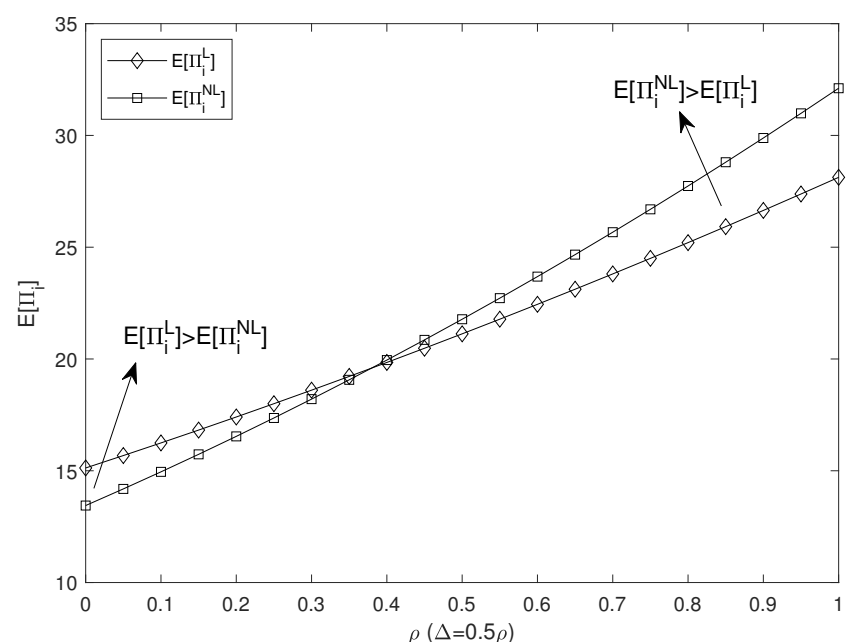

(a) An example of the incumbent's preference when $s=h$.

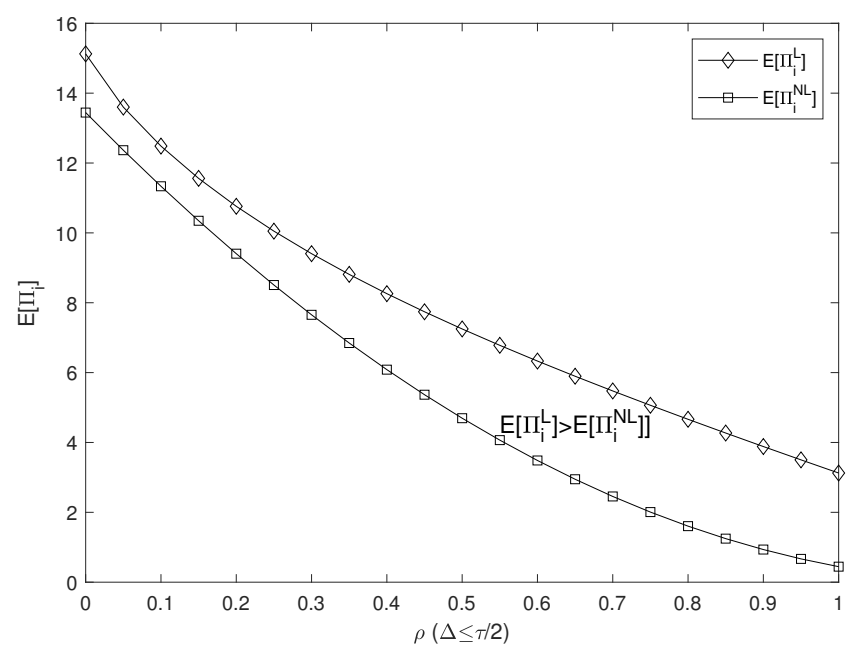

(b) An example of the incumbent's preference when $s=l$ and $\Delta \leq \frac{\tau}{2}$.

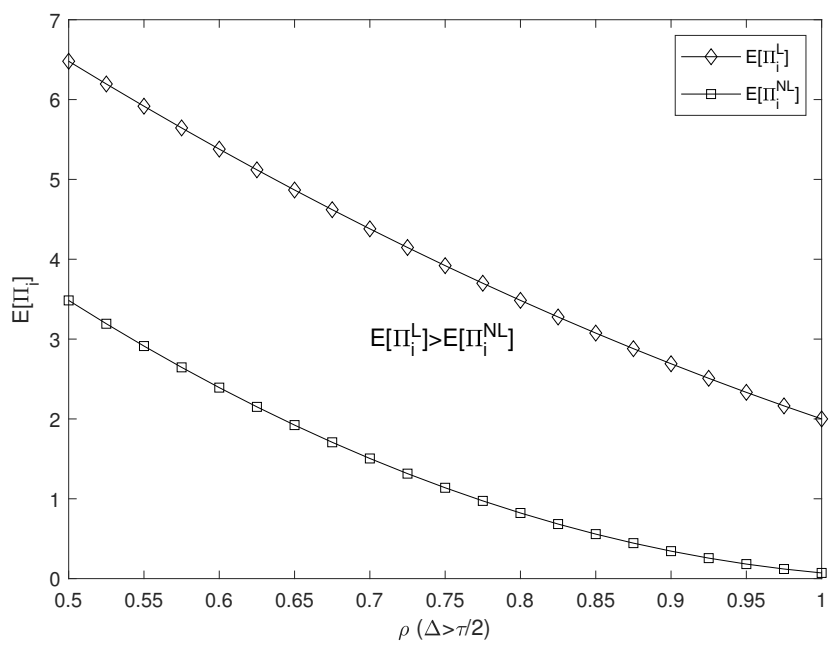

(c) An example of the incumbent's preference when $s=l$ and $\Delta>\frac{\tau}{2}$.

Fig. 2 Example of a three-part figure with individual subcaptions showing that the incumbent prefers information leakage under certain conditions. the incumbent prefers information leakage, which indicates that the preferences of the manufacturer and the incumbent will be inconsistent under such a condition.

When the incumbent forecasts that the market demand will be high (i.e., $s=h$ ), the incumbent's preference is impacted by the probability of high-type market demand $\theta$ and the forecasting accuracy $\rho$. Specifically, if the probability of high-type market demand is high enough, the incumbent's expected profit under information leakage is larger than no information leakage when $s=h$, which means that the incumbent always prefers information leakage in such a case. When $s=h$, if the probability of high-type market demand is not high enough, the incumbent's preference for information leakage is impacted by her forecasting accuracy. In detail, under the condition of the probability of hightype market demand is not high, the incumbent will prefer information leakage if and only if her forecasting accuracy is low. As the Proposition 2 has been indicated, the manufacturer always prefers information leakage when $s=h$, this shows that the information leakage may benefit both the manufacturer and the incumbent simultaneously.

As the examples of Proposition 3, the incumbent's expected profit can be viewed as a function of $\rho$ given $\omega$, $\bar{A}, \delta$ and $\theta$. Similarly, we use the solid line with diamond in Figure 2 to represent the incumbent's expected profit under the scenario that the manufacturer leaks and the solid line with square to represent another scenario. We can find that the incumbent prefers information leakage in some cases when $s=h$, and she will always prefer information leakage when $s=l$.

\subsection{The entrant's performance comparison}

We analyse the influence of the incumbent's inaccurate forecasting to the entrant by comparing his expected profits under different cases in this part. As a competitor of the incumbent, the entrant must be impacted by the incumbent's ordering decision and forecasting information. We list the comparison results in the Proposition 4.

Proposition 4 The result of the entrant's performance comparison is as follows:

(1) When $s=l, \mathrm{E}\left[\Pi_{e}^{N L}\right]>\mathrm{E}\left[\Pi_{e}^{L}\right]$;

(2) When $s=h$, the result is impacted by $\theta$ and $\rho$ : $\circ$ If $\theta \geq \frac{7}{8}, \mathrm{E}\left[\Pi_{e}^{N L}\right]>\mathrm{E}\left[\Pi_{e}^{L}\right]$

- If $\theta<\frac{7}{8}, \mathrm{E}\left[\Pi_{e}^{L}\right]>\mathrm{E}\left[\Pi_{e}^{N L}\right]$ when $\rho>\frac{1-\delta+2 \theta \delta}{6(1-\theta) \delta}$, and vice versa.

From the Proposition 4, if the incumbent forecasts that the market demand will be low (i.e., $s=l$ ), for any 
forecasting accuracy $\rho$ and the probability of high-type market demand $\theta$, the entrant's expected profit under no information leakage is larger than information leakage, which means that leaking the incumbent's order information reflecting private forecasting information is harmful to the entrant when $s=l$. From the Proposition 3, we can find that the two competing retailers' preferences are inconsistent if the incumbent observes a low-type forecast signal.

If the incumbent forecasts that the market demand will be high (i.e., $s=h$ ), the entrant's preference for information leakage is affected by the probability of hightype market demand $\theta$ and the incumbent's forecasting accuracy $\rho$. When the market demand is stable in high-type, knowing the incumbent's order information

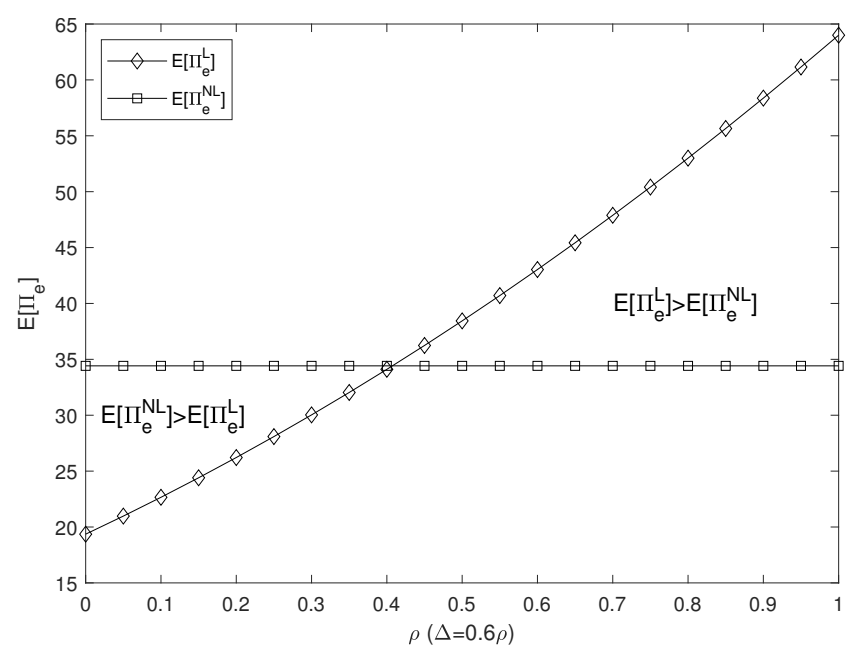

(a) An example of the entrant's performance comparison when $s=h$.

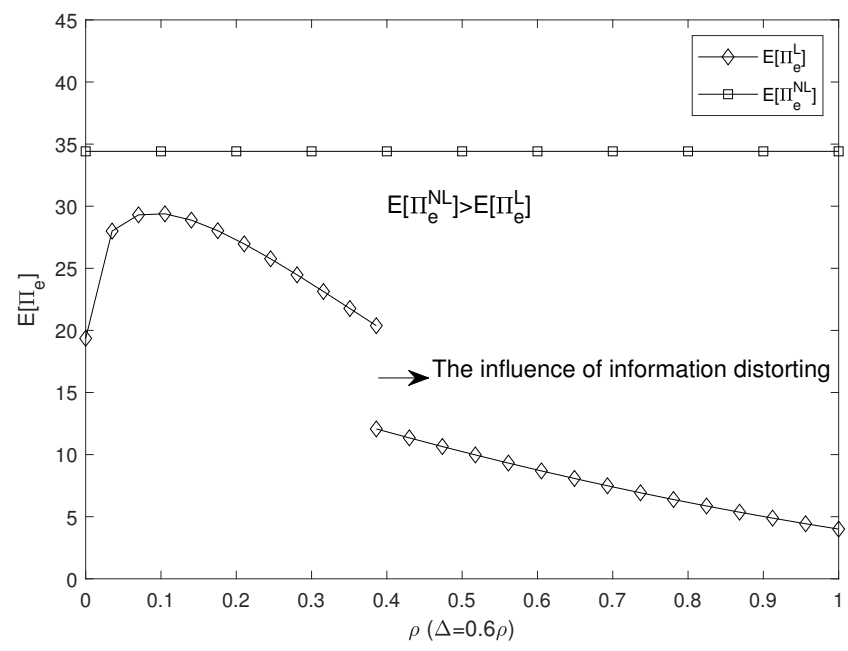

(b) An example of the entrant's performance comparison when $s=l$.

Fig. 3 Example of a two-part figure with individual subcaptions showing that the incumbent's prediction will affect the entrant's preference for information leakage. will hurt his profit for any $\rho$. Once the probability of market demand is high, for any forecasting accuracy, the entrant always prefers no information leakage. But when the probability of high-type market demand is not high, the entrant's preference of information leakage is affected by the incumbent's forecasting accuracy, the entrant prefers information leakage if and only if the incumbent's forecasting accuracy is high enough, otherwise, the entrant will prefer no information leakage for he does not want to undertake the risk of the incumbent's prediction error.

We illuminate the entrant's expected profit comparison under different scenarios in Figure 3. The entrant expected profit can be viewed as a function of $\rho$ for $\omega=0, \bar{A}=20, \delta=0.6$ and $\theta=0.4$. Here, we use the solid line with diamond to represent the entrant's expected profit under the case of information leakage, and the solid line with square represents the scenario of without information leakage. From Figure 3, we can find that the incumbent's prediction will impact the entrant's preference for information leakage.

\section{Conclusions}

The competing relationship and asymmetric forecasting information between Circuit City and Best Buy motivated them to source strategically and manage information carefully. The forecasting information of uncertain demand is often viewed as an important basis of firms' decisions (e.g., Zara and Mimoco) and is asymmetric between retailers (e.g., Circuit City and Best Buy). To investigate retailers' sourcing decisions and supply chain members' information management strategies under such a condition, we developed a framework consisting of an upstream manufacturer and two competing retailers in which only the incumbent retailer can forecast the market demand.

By solving the question of the two retailers' optimal sourcing quantities, we find that, under the condition of competing retailers with asymmetric forecasting information endowment, the incumbent indeed has an incentive to manage her sourcing orders to manage her forecasting information and realize more income if the upstream manufacturer leaks her order information to the entrant. Furthermore, we compare the two retailers' performances under the case of information leakage and no information leakage, thereby, we find some interesting findings: First, for the manufacturer, it prefers information leakage if the incumbent obtains a high-type forecast signal or the incumbent observes a low-type forecast signal and her forecasting accuracy is low, otherwise, the manufacturer would prefer no information 
leakage. Second, both the probability of high-type market demand and the incumbent prediction will affect the incumbent's preference. The incumbent always prefers information leakage when she observes a low-type forecast signal, and the incumbent's preference will be impacted by the probability of high-type market demand and her forecasting accuracy when she observes a hightype forecast signal, specifically, the incumbent will prefer no information leakage if and only if the probability of high-type market demand is not large and her forecasting accuracy is low, otherwise, the incumbent will prefer information leakage. Third, the entrant's preference for information leakage is impacted by the probability of high-type market demand and the incumbent's prediction. If the incumbent's forecast signal is lowtype, the entrant always prefers no information leakage for any forecasting accuracy, but if the incumbent's forecast signal is high-type, the entrant's preference is influenced by the probability of high-type market demand and the incumbent's forecasting accuracy. In detail, if the incumbent forecasts that the market demand will be high, the entrant will prefer no information leakage when the probability of high-type market demand is high or the incumbent's forecasting accuracy is low.

Based on our investigation, some managerial implications are suggested as following: First, from the perspective of the upstream manufacturer's maximum profit, if the incumbent's forecasting signal is $h$, or the incumbent's forecasting signal is $l$ and the incumbent's forecasting accuracy is low, the information leakage strategy should be implemented. Otherwise, the strategy of no information sharing should be implemented. Second, from the perspective of the incumbent's maximum profit, when the incumbent forecasts that the market demand will be low, or she forecasts that the market demand will be high and the market demand is stable in high-type or her forecasting accuracy is low, the information leakage strategy should be implemented. However, when the incumbent forecasts the market demand will be high and her forecasting accuracy is high enough, the no information sharing strategy should be implemented. Third, from the perspective of the entrant's maximum profit, only when the incumbent's forecasting signal is high-type, the market demand is not stable in a high-type and the incumbent accuracy is large enough, the information leakage strategy should be implemented, otherwise, the strategy of no information sharing should be implement.

There are some limitations of the game model we have considered in this study, and some promising questions require further research. We adopt the Cournot game to capture the competition between the two retailers. It would be interesting to explore price compe- tition, which can be modelled, for instance, as Bertrand competition. Additionally, we have compared the supply chain members' performances to find their preferences in response to inaccurate forecasting information in our paper, but the specific coordination contract between the manufacturer and the incumbent retailer in response to the forecasting information is still unclear. These problems are deserving of future study.

\section{Declarations}

Funding Not applicable.

Conflicts of interest All authors have no conflicts of interest.

Availability of data and material All information about this paper is willing to be transparent.

Code availability Not applicable.

Authors' contributions Not applicable.

\section{Supplementary material}

Supplementary material associated with this paper entitled 'Supplementary material'.

\section{References}

Anand KS, Goyal M (2009) Strategic information management under leakage in a supply chain. Manage Sci 55(3):438-452, DOI 10.1287/mnsc.1080.0930

Cederlund JP, Kohli L, Sherer SA, Y Y (2007) How motorola put cpfr into action. Supply Chain Management Rev 11(7):28-35

Chen J, Guo Z (2014) Strategic sourcing in the presence of uncertain supply and retail competition. Prod Oper Manage 23(10):1748-1760, DOI 10.1111/poms. 12078

Chen Y, Ozer O (2019) Supply chain contracts that prevent information leakage. Manage Sci 65(12):56195650, DOI 10.1287/mnsc.2018.3200

Chen YJ, Xiao W (2012) Impact of reseller's forecasting accuracy on channel member performance. Prod Oper Manage 21(6):1075-1089, DOI 10.1111/j. 1937-5956.2012.01339.x

Fisher M, Hammond J, Obermeyer W, Raman A (1994) Making supply meet demand in an uncertain world. Harv Bus Rev 72(3):83-93

Guo X, Xiao G, Zhang F (2017) Effect of consumer awareness on corporate social responsibility under asymmetric information. SSRN Electronic J DOI $10.2139 /$ ssrn.3039862 
Huang S, Xiao W, Yang J (2019) Optimal contracts under endogenous demand information acquisition. Oper Res Lett 47(3):173 - 177, DOI 10.1016/j.orl. 2019.03.004

Iyer G, Narasimhan C, Niraj R (2007) Information and inventory in distribution channels. Manage Sci 53(10):1551-1561, DOI 10.1287/mnsc.1070.0713

Jaber MY, Zanoni S, Zavanella LE (2014) Economic order quantity models for imperfect items with buy and repair options. Int J Prod Econ 155:126 - 131, DOI 10.1016/j.ijpe.2013.10.014

Jain A, Sohoni MG (2015) Should firms conceal information when dealing with common suppliers. Nav Res Log 62(1):1-15, DOI 10.1002/nav.21609

Jiang B, Tian L, Xu Y, Zhang F (2016) To share or not to share: Demand forecast sharing in a distribution channel. Mark Sci 35(5):800-809, DOI 10.1287/mksc. 2016.0981

Jiang B, Sudhir K, Zou T (2020) Effects of costinformation transparency on intertemporal price discrimination. Prod Oper Manage n/a(n/a), DOI 10.1111/poms. 13270

Khalilpourazari S, Pasandideh S, Niaki S (2019) Optimizing a multi-item economic order quantity problem with imperfect items, inspection errors, and backorders. Soft Comput 23(n/a):11671-11698, DOI 10.1007/s00500-018-03718-1

Kim H, Lu JC, Kvam PH, Tsao YC (2011) Ordering quantity decisions considering uncertainty in supplychain logistics operations. Int J Prod Econ 134(1):16 - 27, DOI 10.1016/j.ijpe.2011.02.017

Kong G, Rajagopalan S, Zhang H (2013) Revenue sharing and information leakage in a supply chain. Manage Sci 59(3):556-572, DOI 10.1287/mnsc.1120.1627

Li L (2002) Information sharing in a supply chain with horizontal competition. Manage Sci 48(9):1196-1212, DOI 10.1287/mnsc.48.9.1196.177

Li L, Zhang H (2008) Confidentiality and information sharing in supply chain coordination. Manage Sci 54(8):1467-1481, DOI 10.1287/mnsc.1070.0851

Li Z, Zhang H, Gao R (2020) Frugal innovation in supply chain cooperation considering e-retailer's platform value. Soft Comput 24(n/a):15373-15387, DOI $10.1007 / \mathrm{s} 00500-020-04872-1$

Mailath GJ, Okuno-Fujiwara M, Postlewaite A (1993) Belief-based refinements in signalling games. J Econ Theory 60(2):241 - 276, DOI 10.1006/jeth.1993.1043 Mishra BK, Raghunathan S, Yue X (2007) Information sharing in supply chains: Incentives for information distortion. Iie Trans 39(9):863-877, DOI 10.1080/07408170601019460

Nasser S, Turcic D (2019) Temporary contract adjustment to a retailer with a private demand forecast.
Manage Sci 65(1):209-229, DOI 10.1287/mnsc.2017. 2942

Niu B, Li J, Zhang J, Cheng HK, Tan YR (2019) Strategic analysis of dual sourcing and dual channel with an unreliable alternative supplier. Prod Oper Manage 28(3):570-587, DOI https://doi.org/10.1111/poms. 12938

Taylor TA, Xiao W (2010) Does a manufacturer benefit from selling to a better-forecasting retailer? Manage Sci 56(9):1584-1598, DOI 10.1287/mnsc.1100.1204

Wang Y, Tang W, Zhao R (2019) Supplier's strategy: align with the dominant entrant retailer or the vulnerable incumbent retailer? Soft Comput 23:3481 3500, DOI 10.1007/s00500-018-3008-1

Wu X, Zhang F (2014) Home or overseas? an analysis of sourcing strategies under competition. Manage Sci 60(5):1223-1240, DOI 10.1287/mnsc.2013.1823

Yan Y, Zhao R, Lan Y (2017) Asymmetric retailers with different moving sequences: Group buying vs. individual purchasing. Eur J Oper Res 261(3):903 917, DOI 10.1016/j.ejor.2017.02.025

Zhang H (2002) Vertical information exchange in a supply chain with duopoly retailers. Prod Oper Manage $11(4): 531-546$, DOI 10.1111/j.1937-5956.2002. tb00476.x

Zhu X, Mukhopadhyay SK, Yue X (2011) Role of forecast effort on supply chain profitability under various information sharing scenarios. Int J Prod Econ 129(2):284 - 291, DOI 10.1016/j.ijpe.2010.10.021 
Figures

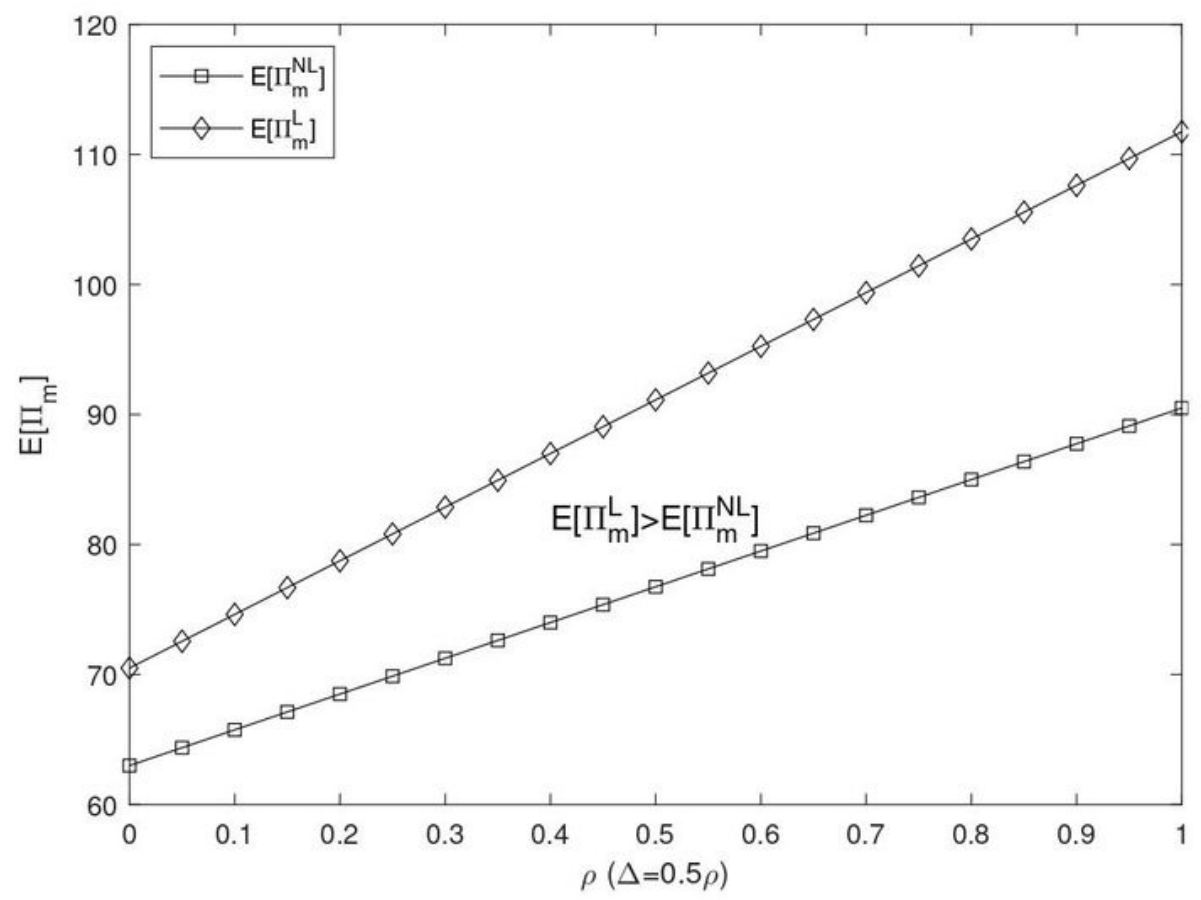

(a) An example of the manufacturer's preference when $s=h$.

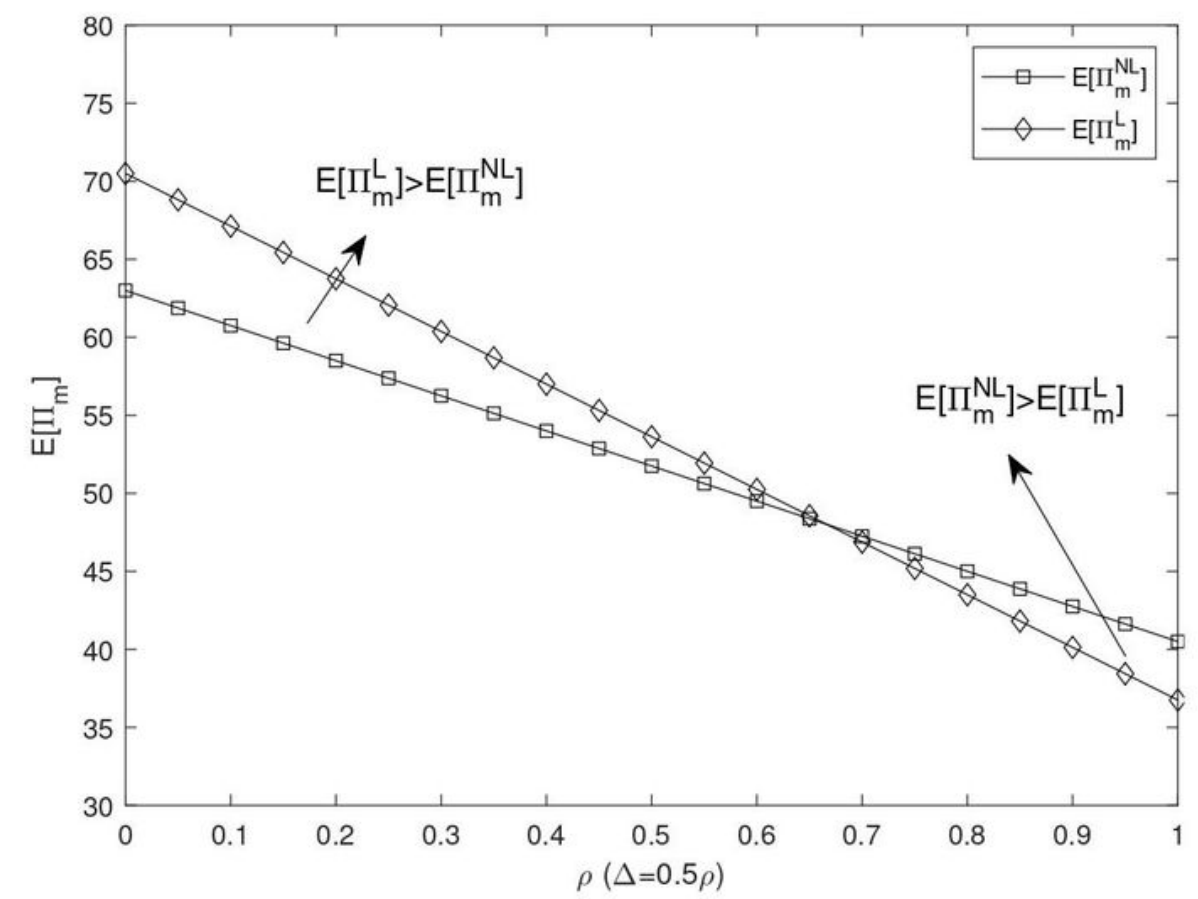

(b) An example of the manufacturer's preference when $s=l$.

\section{Figure 1}

Example of a two-part figure with individual sub-captions showing that information leakage may hurt the manufacturer's profit 


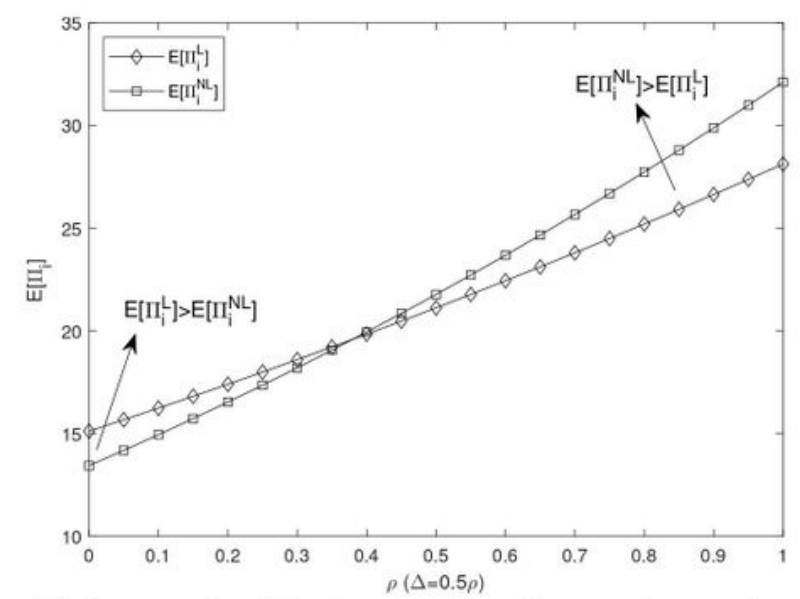

(a) An example of the incumbent's preference when $s=h$.

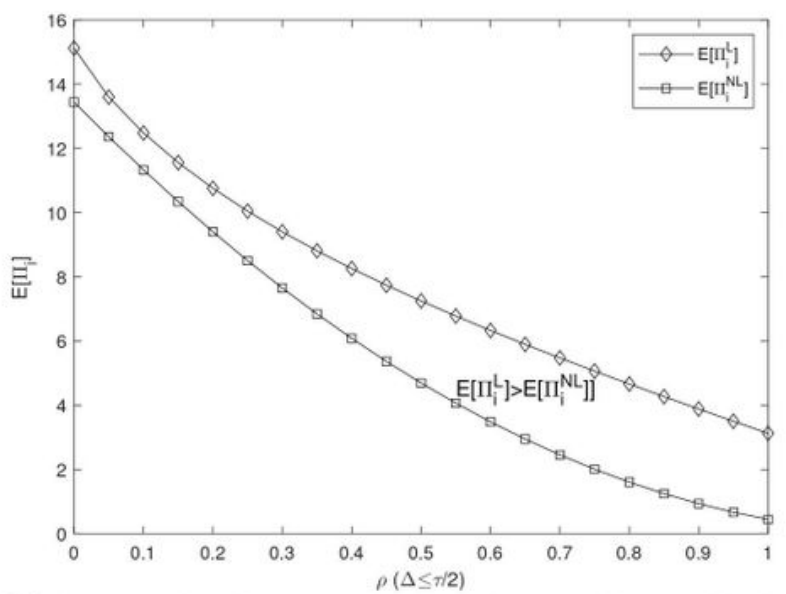

(b) An example of the incumbent's preference when $s=l$ and $\Delta \leq \frac{\tau}{2}$.

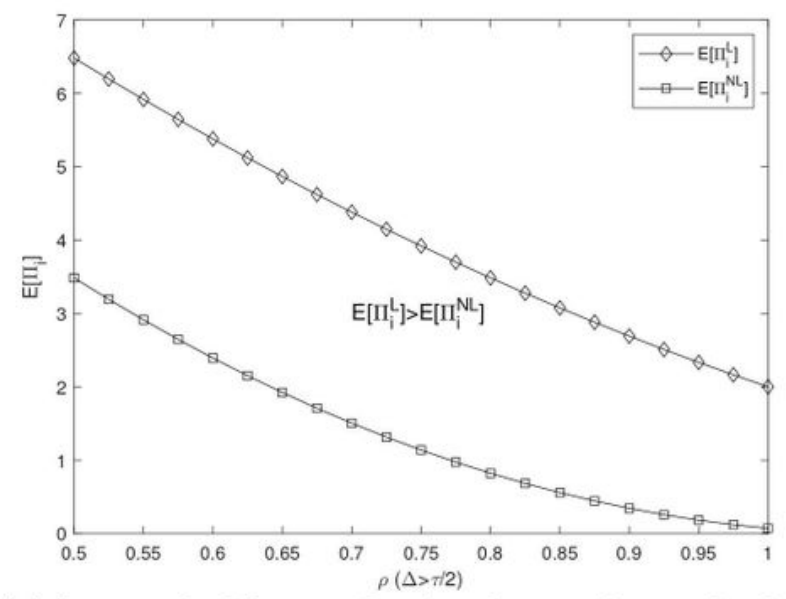

(c) An example of the incumbent's preference when $s=l$ and $\Delta>\frac{\tau}{2}$.

\section{Figure 2}

Example of a three-part figure with individual sub-captions showing that the incumbent prefers information leakage under certain conditions. 


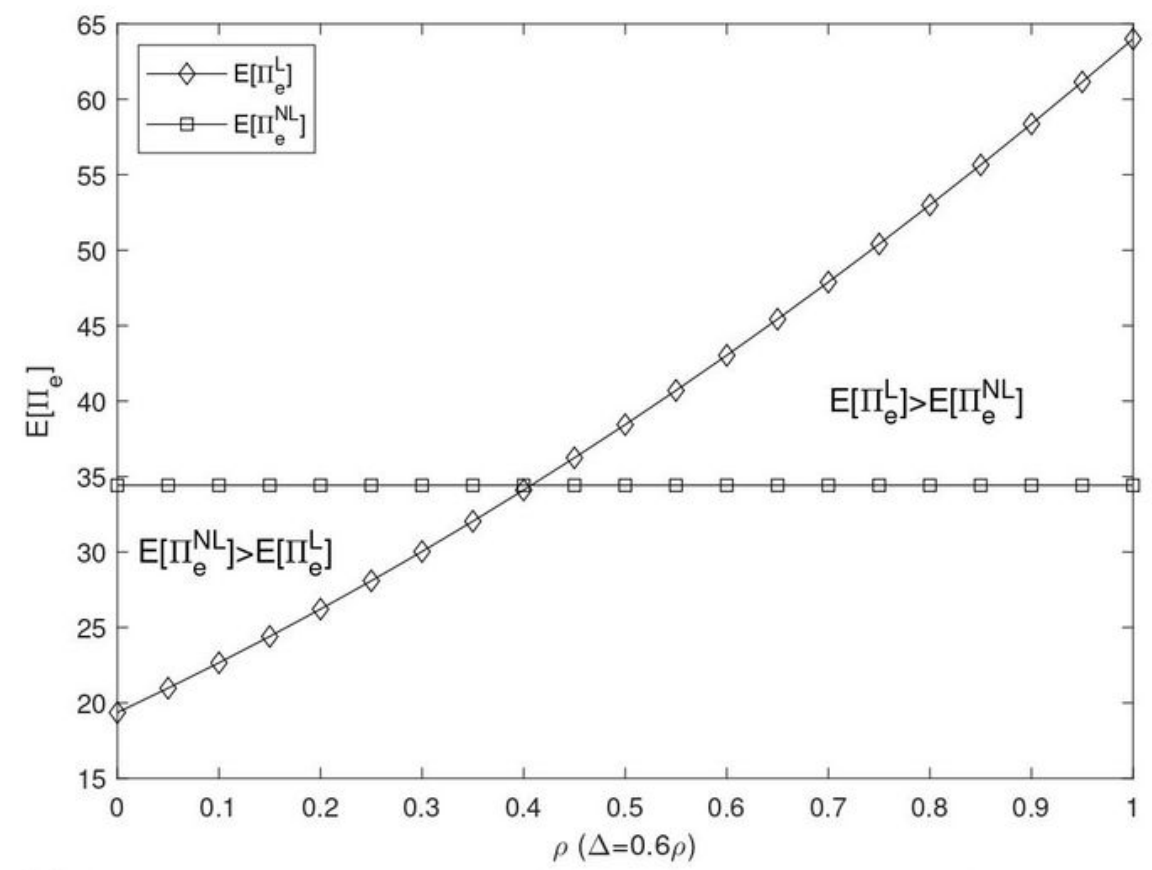

(a) An example of the entrant's performance comparison when $s=h$.

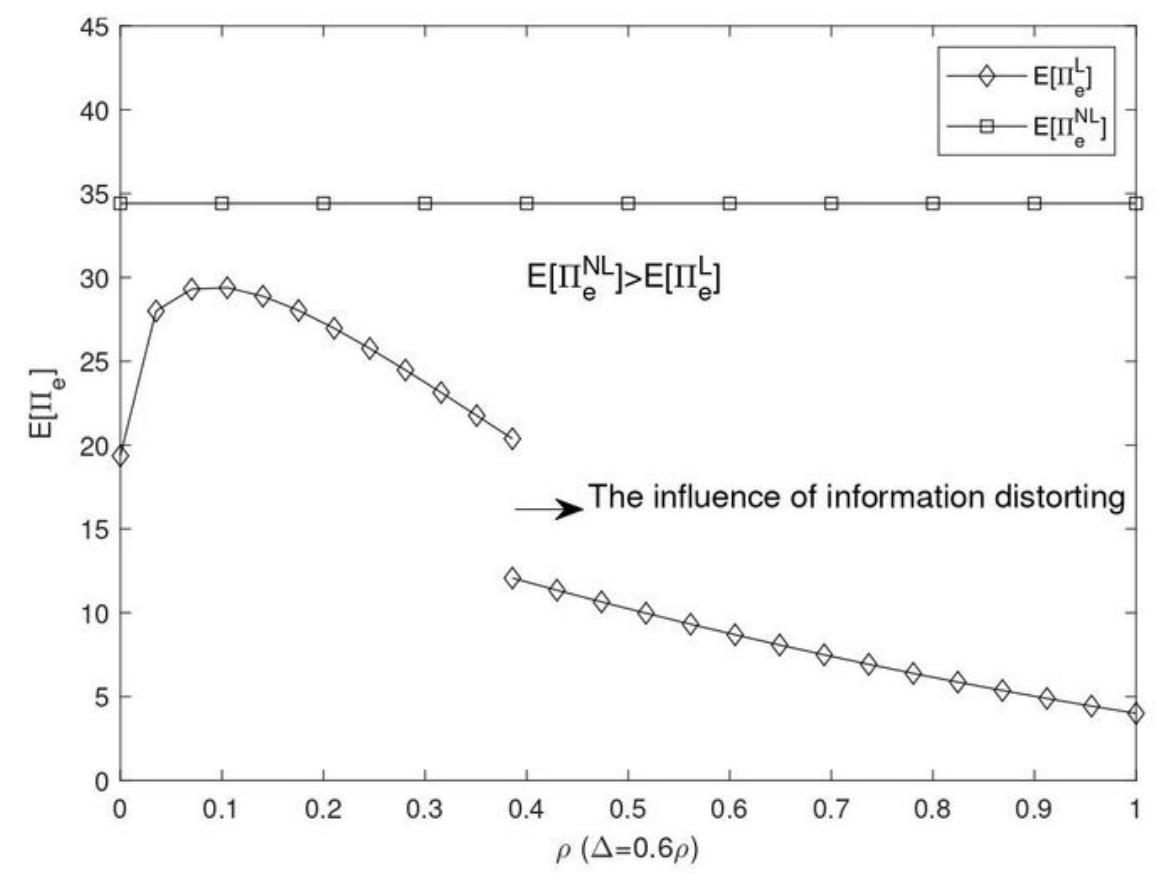

(b) An example of the entrant's performance comparison when $s=l$.

\section{Figure 3}

Example of a two-part figure with individual sub-captions showing that the incumbent's prediction will affect the entrant's preference for information leakage.

\section{Supplementary Files}


This is a list of supplementary files associated with this preprint. Click to download.

- Supplementarymaterial.pdf 